\title{
Predictors of Family Medicine Patient Retention in Opioid Medication-Assisted Treatment
}

\author{
Kathryn Justesen, MD, Stephanie A. Hooker, $\mathrm{PhD}, \mathrm{MPH}$, \\ Michelle D. Sherman, PhD, ABPP, Mary Lonergan-Cullum, PhD, Tanner Nissly, DO, \\ and Robert Levy, $M D$
}

Purpose: Opioid use disorder is a prevalent and chronic condition that can lead to adverse outcomes if untreated. Medication-assisted treatment (MAT) with buprenorphine in a primary care setting has the potential to increase availability of treatment and reduce harm; however, retention in MAT is key for patient success. This study's purpose was to examine predictors of retention in a MAT program for OUD in a family medicine residency clinic.

Methods: A retrospective chart review was conducted for 238 patients diagnosed with OUD and receiving MAT at a family medicine residency clinic between 2015 to 2017 , with visit and prescription data collected through December 2018. Cox-proportional hazards models were used to examine the length of time in treatment.

Results: Over three-fourths of our patients were retained for at least 3 months, $69 \%$ for at least 6 months, and $48 \%$ retained for at least 1 year. Physician continuity of care and having insurance coverage significantly predicted retention and longer duration of treatment.

Conclusions: Continuity of care and having health insurance were key predictors of patient retention in MAT care. Our findings emphasize the clinical significance of maintaining physician continuity of care to improve retention of patients with OUD in MAT programs. Future research could explore what aspects of continuity of care lead to retention in OUD treatment. ( $\mathrm{J}$ Am Board Fam Med 2020;33:848-857.)

Keywords: Buprenorphine, Continuity of Patient Care, Duration of Therapy, Internship and Residency, Family Physicians, Opioid-Related Disorders, Primary Health Care, Proportional Hazards Models, Retrospective Studies

Opioid use disorder (OUD) is a chronic condition well suited to treatment in primary care settings. ${ }^{1}$ Patients with OUD can be treated in primary care settings with limited on-site resources and have similar outcomes to those emerging from specialized treatment centers. ${ }^{2}$ Family medicine physicians are experts in managing chronic conditions and can

This article was externally peer reviewed.

Submitted 1 March 2020; revised 2 June 2020; accepted 7 June 2020.

From the Department of Family Medicine and Community Health North Memorial Family Medicine Residency Program, University of Minnesota, Minneapolis, MN (KJ, MDS, MLC, TN, RL); Research Division, HealthPartners Institute, Minneapolis, $\mathrm{MN}$ (SH).

Funding: Financial support for this project has been received from The Minnesota Department of Human Services State Opioid Response grant. Sponsors did not serve a role in the design, data collection, analysis and interpretation, or manuscript preparation for this study.

Conflict of interest: None.

Corresponding author: Kathryn Justesen, MD, Department of Family Medicine and Community Health, University of offer valuable continuity of care for patients, something which may be difficult to achieve with specialty providers. Family medicine physicians are also well versed in managing complex physical and mental health problems that often accompany OUD. Family medicine residency programs, in particular, are uniquely poised to address the opioid use epidemic by teaching the next generation of family physicians evidence-based skills and providing supervised experience in treating patients with OUD. Implementing medication-assisted treatment (MAT) with buprenorphine within a family medicine residency program serves the clinic's patients and creates a training environment where residents gain knowledge and confidence in providing MAT, ultimately increasing availability of MAT in the community.

Minnesota Medical School, MMC 381, 420 Delaware St. SE, Minneapolis, MN 55455 (E-mail: gate0051@umn.edu). 
Improving retention in MAT programs is paramount to addressing the opioid epidemic, and continued engagement in MAT is key for improving patient outcomes. Retention in care is associated with improved mortality and morbidity, making it a quality measure for OUD treatment programs. ${ }^{3}$ Retaining patients in MAT programs has proven difficult, with 6-month retention rates ranging between 33 to $66 \%$ and most studies reporting approximately $50 \%$ retention across most MAT settings. ${ }^{4-6}$ Several key variables have consistently shown positive correlations with retention, including older age, ${ }^{5,7-12}$ female gender, ${ }^{12,13}$ receiving outpatient psychotherapy, ${ }^{5}$ and engagement in a contingency management program. ${ }^{4}$ Identifying as African-American or Hispanic/Latino, having lower education level, and being single have been associated with a lower rate of retention. ${ }^{5,7-9,12-15}$ Using illicit substances during treatment predicts early dropout. $^{9,10}$ Martin and colleagues ${ }^{3}$ provide a recent comprehensive summary of patient characteristics predicting retention. There is a lack of agreement within the literature as well as no clear definition put forth by the American Society of Addiction Medicine for "retention." Other studies have utilized sobriety as their outcome rather than simply retention ${ }^{2}$, however this does not allow for the fact that OUD is a chronic disease of which relapse is a component. For the purposes of our study, we utilized a visit-based definition of retention, which is one of the most commonly used measures of retention in the literature..$^{3,5,7,13}$

Predictors of MAT program retention in a family medicine residency clinic have not been thoroughly investigated. Previous studies examining predictors of retention have been conducted in environments other than family medicine residency clinics. The family medicine residency clinic is a critical environment for training physicians to identify and treat OUD as a chronic disease. However, it can also be a challenging environment to implement addiction treatment as residents have varying schedules and are not as frequently in clinic as fulltime practicing physicians. Understanding patient characteristics and treatment retention in this setting is key for delivering optimal patient care. The current study aims to 1) describe patients who received MAT in a family medicine residency training clinic, and 2) explore predictors of retention in a residencybased MAT program. Improving our understanding of factors related to retention can guide clinical initiatives to support patients at higher risk for relapse or dropout.

\section{Methods \\ Setting}

Broadway Family Medicine is a University of Minnesota Department of Family Medicine and Community Health residency training clinic. The clinic is located in a socioeconomically depressed area of North Minneapolis. The clinic started providing MAT in 2011, with 1 physician prescribing buprenorphine. Currently, all 10 of the core faculty physicians plus 16 resident physicians have their DATA 2000 waivers to prescribe buprenorphine according to the Drug Addiction and Treatment Act of 2000. In addition, the clinic has 2 full-time psychologists.

Patients participating in the MAT program are initially in the Substance Use Recovery Clinic, a clinic session provided once a week within the family medicine clinic and staffed by a rotation of resident physicians. The only defined exclusion criterion for receiving buprenorphine at our clinic is current failure of treatment within a methadone program, evidenced by still being on daily take-out prescriptions after 1 year of methadone treatment. Subsequent visits for buprenorphine prescriptions are fully integrated into providers' primary care clinic schedules. On induction, patients receiving buprenorphine prescriptions are seen 1 to 2 times weekly for 3 weeks, then every 2 weeks for 4 weeks, then monthly for 1 year. Priority is placed on having these follow-up visits consistently with 1 primary provider; however, due to the nature of resident physicians' schedules this is not always possible. After 1 year, consideration is given to extending visits to every 2 or 3 months, depending on a variety of criteria. A urine drug screen is performed at every visit.

\section{Participants and Procedure}

This study used a retrospective chart review design. Patients who were seen by a physician at Broadway Family Medicine between January 2015 and December 2017, were given at least 1 prescription of buprenorphine, and had a diagnosis of "opioid use disorder on agonist therapy" in their problem list were included in the study sample. Patients are allowed to return to treatment at clinic after a lapse in care except for rare instances that involved verbal or physical abuse toward a staff member or provider.

Variables of interest were extracted from the electronic health record (Epic; see Measures for a desc- 
ription of the variables). Visit and prescription data were extracted through the end of December 2018. In addition, manual chart reviews were conducted to determine whether patients were new inductions or continuations at the start of treatment, the reasons that patients discontinued from the program, and whether patients who died during the study period were actively engaged in the program. The University of Minnesota Institutional Review Board reviewed the study protocol and deemed the study as exempt (IRB \#STUDY00003127).

\section{Measures}

\section{Retention and Duration of MAT Treatment}

The primary outcome variable was retention in the MAT program, defined by having a visit with a primary care provider (PCP) in December 2018 or having a prescription for buprenorphine that extended past the last day of the study period (December 31, 2018). The proportion of patients who were retained at 3 months, 6 months, and 12 months was also calculated. The total duration of treatment was the number of days between the first date and the last date that patients received a buprenorphine prescription. This definition of retention allows for lapses and gaps in care. In addition, patients who died while they were still active in the MAT program were considered "retained" (censored); those who discontinued treatment before their death were considered "dropped."

\section{Sociodemographic Characteristics}

Sociodemographic characteristics included age (age on the date of first buprenorphine prescription), sex, race/ethnicity (white, black, or other), marital status, and insurance type (Medicaid, Medicare, private insurance, or self-pay).

\section{Clinic Visits}

Engagement with the MAT program was assessed via counts of the number of total completed visits with a PCP for OUD treatment and for other concerns. In addition, the total number of behavioral health visits during this time period was also gathered. Because a minority of patients had any contact with a behavioral health provider, the number of visits was recoded to a dichotomous variable ( $1=$ seen by behavioral health; $0=$ not seen by behavioral health).

\section{Continuity of Care}

Continuity of care was calculated using the K-index, a measure of multiple provider continuity. ${ }^{16-18}$ The $\mathrm{K}$-index is calculated by:

$$
\frac{(n-i)}{(n-1)}
$$

where $\mathrm{n}$ equals the total number of PCP visits and $\mathrm{i}$ equals the number of different physicians seen. The calculated index score ranges from 0 to 1 , with 0 demonstrating no continuity of care (all visits were performed by different PCPs) and 1 demonstrating perfect continuity of care (all visits were performed by the same PCP).

\section{Comorbid Mental Health Disorders and Psychoactive Medications}

Mental health diagnoses were coded based on International Classification of Diseases, 10th Edition (ICD-10) codes. Diagnoses had to be on the patient's problem list in December 2018 in Epic to be included in the list. These diagnoses included depressive disorders, anxiety disorders, bipolar disorders, schizophrenia and other psychotic disorders, posttraumatic stress disorder, attention deficit hyperactivity disorder, and personality disorders. In addition, prescriptions for mental health disorders were obtained to examine whether treatment of comorbid mental health disorders impacted retention. Prescriptions were categorized by type, including antidepressants (selective serotonin reuptake inhibitors [SSRIs] and selective norepinephrine reuptake inhibitors [SNRIs]), antipsychotics, mood stabilizers, benzodiazepines, attention deficit hyperactivity disorder medications and stimulants, and other antidepressants.

\section{Data Analysis}

Data were analyzed using SAS version 9.4 (SAS Institute, Cary, NC). Descriptive statistics (means and SDs for continuous variables and frequencies and percentages for categorical variables) were calculated. Patients were categorized as "retained" or "dropped" at 3 months, 6 months, 12 months, and at the end of the study observation period (December 2018). Fisher exact tests (for categorical variables with cells $<5$ ), $c^{2}$ analyses (for categorical variables), and independent samples $t$-test (for continuous variables) were used to examine statistical differences between the groups on each demographic and clinical characteristic. A $\mathrm{Z}$ transformation was used to aid in 
interpretability of the continuity of care score in the survival model.

A Cox Proportional Hazards model was used to predict treatment retention. If patients discontinued MAT treatment during the study period, they were coded as "dropped." Patients who had received a prescription for buprenorphine that extended past the last day of the study observation period (December 31, 2018) were coded as "retained" and were censored in the model. In addition, patients who died during the study observation period and were actively engaged in MAT treatment were censored in the final analysis. The model included basic demographic characteristics (age, gender, race, insurance status); clinical and visit characteristics were included in the final model if they were significantly related to retention in the bivariate analyses. Of note, 5 patients only had 1 visit during the study observation period and thus could not have a continuity of care score calculated. An additional 2 patients were missing smoking status. These 7 patients were excluded from the final analysis $(n=231)$. In addition, we conducted a sensitivity analysis excluding patients who had tapered off buprenorphine or who had transferred their care to another clinic from the survival model to determine whether the same variables remained predictors of treatment retention.

\section{Results}

Over the course of these 3 calendar years (2015 to 2017), our clinic prescribed buprenorphine to 238 unique patients living with OUD. Eleven patients died during the study period (4.6\%); 6 were actively involved in the MAT program at the time of death (55\%). Four died of opioid overdose, and 1 died from diabetes complications; the remaining causes of death were not confirmed in the electronic health record (EHR). At the first visit, 142 patients $(60 \%)$ were continuing buprenorphine after starting it elsewhere, 41 patients (17\%) were restarting after discontinuing, and 55 (23\%) were new inductions. Of the continuations, most received buprenorphine from a different clinic $(\mathrm{n}=101,71 \%)$, yet some received it from friends or family members $(\mathrm{n}=12,8 \%)$ and others bought it on the street $(n=29,20 \%)$. Across the 4-year observation period (2015 to 2018), patients received an average of 17.0 ( $\mathrm{SD}=14.4$; range, 1 to 78 ) buprenorphine prescriptions and had an average of 20.3 visits with PCPs $(\mathrm{SD}=16.8)$ with an average of 7 different providers
$(\mathrm{SD}=5.3)$. Buprenorphine was ordered in a majority of the patients' visits to primary care ( $M=80 \%$; $\mathrm{SD}=25 \%$ ). Approximately $20 \%$ of patients had at least 1 visit with a behavioral health provider during the 4-year study observation period.

Demographic, clinical, and visit characteristics of the sample are presented in Table 1. The majority of patients were in their mid-30s, single/never married, and white $(71 \%)$, followed by black (19\%). The most common comorbid conditions at baseline were anxiety (42\%) and depression (34\%). Approximately $39 \%$ of patients were prescribed a SSRI/SNRI, and three-quarters of the patients were current smokers.

\section{Retention}

Across the 4-year observation period, patients were retained for an average of 430 days $(\mathrm{SD}=388$ days; median, 341 days; range, 0 to 1445 ), with $77 \%$ of patients retained for at least 3 months, $69 \%$ of patients retained for at least 6 months, and $48 \%$ of patients retained for 12 months or more. Approximately 34\% of patients were retained at the end of the study observation period (in December 2018). Chart reviews were conducted to determine whether the reason for discontinuation could be determined. For half of the patients, the reason for discontinuation was unclear $(n=77$; $49 \%$ ); for others, there was evidence that patients had relapsed $(n=44 ; 28 \%)$, tapered $(n=15 ; 9.6 \%)$, or transferred care to another clinic $(\mathrm{n}=27 ; 17 \%)$.

Table 2 presents the proportion of patients with each characteristic who were retained or dropped out at the end of the study period. Of the demographic characteristics, bivariate analyses revealed that sex, marital status, and race/ethnicity were not significantly related to retention ( $p s>0.05$ ). Age was significantly related to retention, with patients who were retained being slightly older than those who dropped. Insurance coverage was also significantly related to retention. All patients who were self-pay or uninsured were dropped at the end of the study period, whereas patients with Medicaid and other insurances were more likely to be retained. Patients with anxiety and chronic pain were more likely to be retained; however, none of the remaining comorbid psychiatric disorders or psychoactive medications were related to retention at the end of the study period $(p s>0.05)$. Smoking status was significantly related to retention. Patients who were nonsmokers and former smokers were more likely to be retained than current smokers. 
Table 1. Demographic and Clinical Characteristics of Patients Receiving Buprenorphine in a Family Medicine Residency Clinic $(n=238)$ between January 2015 and December 2017

\begin{tabular}{|c|c|}
\hline Variable & $\mathrm{n}[\%]$ \\
\hline Age, years, mean (SD) & $35.5(11.0)$ \\
\hline Female & $98[41]$ \\
\hline Marital status & $\mathrm{n}=235$ \\
\hline Single, never married & $188[80]$ \\
\hline Married & $38[16]$ \\
\hline Divorced, separated, or widowed & $9[4]$ \\
\hline \multicolumn{2}{|l|}{ Race } \\
\hline Black & $46[19]$ \\
\hline White & $168[71]$ \\
\hline Other & $24[10]$ \\
\hline \multicolumn{2}{|l|}{ Insurance coverage } \\
\hline Medicaid & $139[58]$ \\
\hline Other insurance & $80[34]$ \\
\hline Self-pay/uninsured & $19[8]$ \\
\hline \multicolumn{2}{|l|}{ Comorbid conditions } \\
\hline ADHD & $27[11]$ \\
\hline Anxiety & $101[42]$ \\
\hline Bipolar & $14[6]$ \\
\hline Chronic pain & $34[14]$ \\
\hline Depression & $82[34]$ \\
\hline Personality disorder & $10[4]$ \\
\hline Psychosis & $6[3]$ \\
\hline PTSD & $19[8]$ \\
\hline \multicolumn{2}{|l|}{ Medications } \\
\hline ADHD/stimulants & $18[8]$ \\
\hline Antipsychotics & $36[15]$ \\
\hline Benzodiazepines & $6[3]$ \\
\hline Mood stabilizers & $15[6]$ \\
\hline SSRI/SNRI & $92[39]$ \\
\hline Other antidepressants & $41[17]$ \\
\hline Smoking status & $\mathrm{n}=236$ \\
\hline Current smoker & $175[74]$ \\
\hline Former smoker & $30[13]$ \\
\hline Never smoker & $31[13]$ \\
\hline Number of PCP visits, mean (SD) & $20.3(16.8)$ \\
\hline Number of buprenorphine visits, mean (SD) & $15.6(13.6)$ \\
\hline Number of different PCPs seen, mean (SD) & $7.0(5.3)$ \\
\hline Continuity of care (K index) & $0.64(0.21)$ \\
\hline Seen by behavioral health & $47[20]$ \\
\hline
\end{tabular}

ADHD, attention deficit hyperactivity disorder; PCP, primary care provider; PTSD, posttraumatic stress disorder; SNRI, selective norepinephrine reuptake inhibitor; SSRI, selective serotonin reuptake inhibitor; SD, standard deviation.

Continuity of care was also significantly related to retention, $\mathrm{t}(230.46)=-3.87 ; P<.001$. Patients who were retained had greater continuity of care than patients who discontinued treatment. In addition, patients who saw behavioral health at least 1 time were more likely to be retained ( $n=23$ of 47; $49 \%$ retained) than patients who never saw behavioral health $(=59$ of $191 ; 31 \%$ retained, $\chi 2$ $(1)=5.44 ; P=.02)$. Further examination of behavioral health visits revealed that patients saw behavioral health providers a median of 1 time $(M=2.4$; $\mathrm{SD}=2.9$; range, 1 to 18$)$. Less than half the visits addressed addiction specifically (42\%); other concerns included comorbid mental health conditions (39\%) and care coordination (15\%). Relatively few patients were seen for ongoing psychotherapy $(\mathrm{n}=8 ; 3 \%)$, beyond an initial intake and diagnostic assessment.

\section{Multivariate Analysis}

A Cox proportional hazards model was used to predict treatment retention (Table 3). Controlling for other covariates, insurance type, $\chi 2(2)=16.99$; $P<.001$, and continuity of care, $\chi^{2}(1)=25.61$; $P<.001$, were significantly related to discontinuing treatment. Patients who were self-pay or uninsured had 3.32 times increased risk (95\% CI, 1.86-5.92) of discontinuing treatment compared with patients with Medicaid. Those who were self-pay or uninsured had a 2.40 increased risk (95\% CI, 1.29-4.45) of terminating treatment compared with those with other types of insurance. There were no differences between patients with Medicaid and patients with other types of insurance. Further, a 1-SD increase in continuity of care was associated with a reduced risk of terminating treatment $($ Hazard Ratio $(H R)=$ 0.61; 95\% CI, 0.5-0.74; Figure 1). After including covariates in the model, age, $\chi^{2}(1)=3.70 ; P=.055$, and seeing behavioral health were marginally related to treatment retention, $\chi^{2}(1)=3.84$; $P=.050$. For every 1 -year increase in age, patients had a $2 \%$ decreased risk of dropping out of treatment. Patients who had seen a behavioral health therapist in our clinic had a reduced risk of terminating treatment $(H R=0.61 ; 95 \%$ CI, 0.39-0.93) compared with patients who had never seen behavioral health. Other covariates (gender, race, smoking status, anxiety, and chronic pain) were not significantly related to time in treatment.

\section{Sensitivity Analysis}

In a sensitivity analysis excluding patients who had tapered or transferred their care (model $\mathrm{n}=191$ ), the pattern of results observed in the original model remained similar. Continuity of care $(H R=0.63$; 
Table 2. Demographic and Clinical Characteristics of Patients Receiving Buprenorphine (January 2015 to December 2017) Related to Retention in December 2018 ( $n=238)$

\begin{tabular}{|c|c|c|c|c|c|c|}
\hline \multirow{2}{*}{$\frac{\text { Variable }}{\text { Age, years, mean (SD) }}$} & \multicolumn{2}{|c|}{$\begin{array}{c}\text { Retained } \\
\mathrm{N}=82 \\
\mathrm{n}[\%]\end{array}$} & \multicolumn{2}{|c|}{$\begin{array}{l}\text { Dropped } \\
\mathrm{N}=156 \\
\mathrm{n}[\%]\end{array}$} & \multirow{2}{*}{$\frac{\chi^{2} \text { ort }}{-1.98}$} & \multirow{2}{*}{$\begin{array}{c}P \\
.049\end{array}$} \\
\hline & 37.4 & $(11.5)$ & 34.4 & $(10.6)$ & & \\
\hline Female & 40 & [49] & 58 & {$[37]$} & 2.99 & .08 \\
\hline Marital status & & & & & & .66 \\
\hline Single, never married & 63 & {$[78]$} & 125 & {$[81]$} & & \\
\hline Married & 14 & {$[17]$} & 24 & {$[16]$} & & \\
\hline Divorced, separated, or widowed & 4 & {$[5]$} & 5 & {$[3]$} & & \\
\hline Race & & & & & 5.60 & .06 \\
\hline Black & 9 & {$[11]$} & 37 & {$[24]$} & & \\
\hline White & 64 & {$[78]$} & 104 & {$[67]$} & & \\
\hline Other & 9 & {$[11]$} & 15 & {$[10]$} & & \\
\hline Insurance coverage & & & & & & $<.001$ \\
\hline Medicaid & 51 & {$[62]$} & 88 & {$[56]$} & & \\
\hline Other insurance & 31 & {$[38]$} & 49 & {$[32]$} & & \\
\hline Self-pay/uninsured & 0 & {$[0]$} & 19 & {$[12]$} & & \\
\hline \multicolumn{7}{|l|}{ Comorbid conditions } \\
\hline ADHD & 11 & {$[13]$} & 16 & {$[10]$} & 0.55 & .47 \\
\hline Anxiety & 42 & {$[51]$} & 59 & {$[38]$} & 3.95 & .047 \\
\hline Bipolar & 6 & [7] & 8 & {$[5]$} & 0.47 & .50 \\
\hline Chronic pain & 17 & {$[21]$} & 17 & {$[11]$} & 4.25 & .039 \\
\hline Depression & 31 & {$[38]$} & 51 & {$[33]$} & 0.62 & .43 \\
\hline Personality disorder & 5 & {$[6]$} & 5 & {$[3]$} & 1.12 & .29 \\
\hline Psychosis & 3 & {$[4]$} & 3 & {$[2]$} & & .42 \\
\hline PTSD & 6 & [7] & 13 & {$[8]$} & 0.08 & .78 \\
\hline \multicolumn{7}{|l|}{ Medications } \\
\hline ADHD/Stimulants & 8 & {$[10]$} & 10 & {$[6]$} & 0.86 & .35 \\
\hline Antipsychotics & 12 & {$[15]$} & 23 & {$[15]$} & 0.00 & .98 \\
\hline Benzodiazepines & 2 & {$[2]$} & 4 & {$[3]$} & & 1.00 \\
\hline Mood stabilizers & 6 & [7] & 9 & {$[6]$} & 0.21 & .64 \\
\hline SSRI/SNRI & 28 & {$[34]$} & 64 & {$[41]$} & 1.07 & .30 \\
\hline Other antidepressants & 17 & {$[21]$} & 24 & {$[15]$} & 1.08 & .30 \\
\hline Smoking status $(\mathrm{n}=237)$ & & & & & 11.40 & .003 \\
\hline Current smoker & 50 & {$[60]$} & 125 & {$[81]$} & & \\
\hline Former smoker & 16 & {$[20]$} & 14 & [9] & & \\
\hline Never smoker & 16 & {$[20]$} & 15 & {$[10]$} & & \\
\hline Continuity of care, mean (SD) & 0.70 & $(0.12)$ & 0.61 & $(0.24)$ & -3.87 & $<.001$ \\
\hline Seen by behavioral health & 23 & {$[28]$} & 24 & {$[15]$} & 5.44 & .019 \\
\hline Buprenorphine status at first visit & & & & & 2.16 & .34 \\
\hline New induction & 17 & {$[21]$} & 38 & {$[24]$} & & \\
\hline Restart & 11 & {$[13]$} & 30 & {$[19]$} & & \\
\hline Continuation & 54 & {$[66]$} & 88 & {$[57]$} & & \\
\hline
\end{tabular}

ADHD, attention deficit hyperactivity disorder; PTSD, posttraumatic stress disorder; SSRI, selective serotonin reuptake inhibitor; SNRI, selective norepinephrine reuptake inhibitor; SD, standard deviation.

Independent samples $t$-test were used to examine differences in continuous variables (signified by mean, SD) by retention status; $\chi^{2}$ tests were used to examine differences in categorical variables by retention status. The retained category includes 6 patients who died during the study observation period and are censored in the survival analysis. For variables with cells $<5$, a Fisher's exact test was used to calculate the $P$-value (marital status, insurance type, benzodiazepine prescription, and psychosis). For tables larger than $2 \times$ 2, SAS uses a Monte Carlo simulation to estimate the $P$-value in Fisher's exact tests. 
Table 3. Cox Proportional Hazards Model with Demographic and Clinical Characteristics of Patients Receiving Buprenorphine Predicting Time in Treatment $(\mathrm{n}=231)$ between January 2015 and December 2018

\begin{tabular}{lll}
\hline Variable & HR & $95 \%$ CI \\
\hline Age & 0.98 & {$[0.97,1.00]$} \\
Gender & 0.75 & {$[0.51,1.09]$} \\
Race & & \\
$\quad$ White & REF & \\
$\quad$ Black & 1.54 & {$[0.99,2.41]$} \\
$\quad 1.17$ & {$[0.64,2.12]$} \\
$\quad$ Other & & \\
Insurance status & REF & \\
$\quad$ Medicaid & 1.39 & {$[0.94,2.05]$} \\
$\quad$ Other insurance & 3.32 & {$[\mathbf{1 . 8 6 , 5 . 9 2}]$} \\
$\quad$ Self-pay/uninsured & 0.86 & {$[0.60,1.22]$} \\
Anxiety & 1.18 & {$[0.69,2.02]$} \\
Chronic pain & & \\
Smoking status & REF & \\
$\quad$ Current smoker & 0.59 & {$[0.32,1.07]$} \\
$\quad$ Former smoker & 0.65 & {$[0.37,1.13]$} \\
$\quad$ Never smoker & $\mathbf{0 . 6 1}$ & {$[\mathbf{0 . 5 1}, \mathbf{0 . 7 4}]$} \\
Continuity of care & 0.63 & {$[0.40,1.00]$} \\
Seen by behavioral health &
\end{tabular}

HR, hazard ratio; CI, confidence interval; REF, reference. Bold values are statistically significant at $P<.05$.

95\% CI, 0.50-0.78) and being self-pay or uninsured $(H R=2.44 ; 95 \%$ CI, 1.15-5.19) remained significant predictors of treatment length. Smoking status emerged as a significant predictor of discontinuing treatment, with never smokers less likely to discontinue than current smokers $(H R=0.46$; 95\% CI, 0.22 0.97). Seeing behavioral health was no longer marginally related to treatment discontinuation $(H R=0.65$; 95\% CI, 0.39-1.08). However, age emerged as a significant predictor, with younger patients being more likely to discontinue treatment $(H R=0.97 ; 95 \% \mathrm{CI}$, 0.95-0.99).

\section{Discussion}

This retrospective study of 238 unique patients receiving MAT in a family medicine residency clinic revealed better retention at 6 months $(68.8 \%)$ than rates reported from other buprenorphine treatment programs in community and primary care settings (range, 33\% to $66 \%){ }^{4}$ Slightly less than half $(48 \%)$ of our patients were retained at 1 year, a rate similar to published findings. ${ }^{4,12}$ Consistent with previous research, key predictors of retention included insurance coverage ${ }^{2}$ and continuity of care.

A unique finding in our study is the positive correlation between continuity of care and retention in MAT. It is well known in Family Medicine that continuity of care leads to improved control of chronic diseases such as type 2 diabetes $^{24}$ and that patient satisfaction is positively correlated with continuity of care. ${ }^{5}$ Patients with mental health difficulties also benefit from greater continuity of care. $^{25}$ The finding that continuity of care predicts retention in MAT is compelling. From a relational perspective, this intuitively makes sense, as continuity of care promotes trust and shared responsibility between patients and practitioners. ${ }^{26}$ Although continuity of care can be challenging to achieve in a residency training program, it seems to be related to higher levels of patient retention in MAT. Continuity of care is linked to improved preventative care, reduced hospitalizations and emergency department visits, reduced health care costs, and higher patient satisfaction, and therefore should continue to be prioritized in modern medicine, including addiction medicine. ${ }^{27,28}$ As experts in the management of chronic disease, and with our emphasis on continuity of care, family medicine physicians are ideal providers of MAT.

Consistent with previous research, insurance status predicts retention in MAT programs. ${ }^{2}$ We also replicated an association between smoking status and MAT retention, with current smokers being less likely to be retained in MAT. ${ }^{29}$ A practical implication for family medicine physicians is to encourage their patients in MAT to abstain from smoking, noting the potential benefits for their physical health and their recovery from OUD.

Research on the impact of behavioral health interventions for MAT retention in primary care has been mixed, with many studies finding no significant contribution of behavioral health beyond that offered by MAT., 13,19-21 These studies are often randomized controlled trials in which counseling services are compulsory, ${ }^{20-22}$ and patients treated for OUD report a preference for voluntary access to behavioral health. ${ }^{23}$ In this chart review, we found higher rates of retention among patients who saw a behavioral health provider; this relationship was no longer statistically significant in a multivariable model that included diagnoses of anxiety and chronic pain, 2 conditions for which patients in our clinic may seek behavioral health treatment. 
Figure 1. Survival curve of dropout for patients receiving buprenorphine separated by high, medium, and low continuity of care $(n=232)$ between January 2015 and December 2018. Note. To further understand the impact of continuity of care on retention, survival curves were plotted for patients at high (1 standard deviation above the mean), medium (at the mean), and low ( 1 standard deviation below the mean) continuity of care.

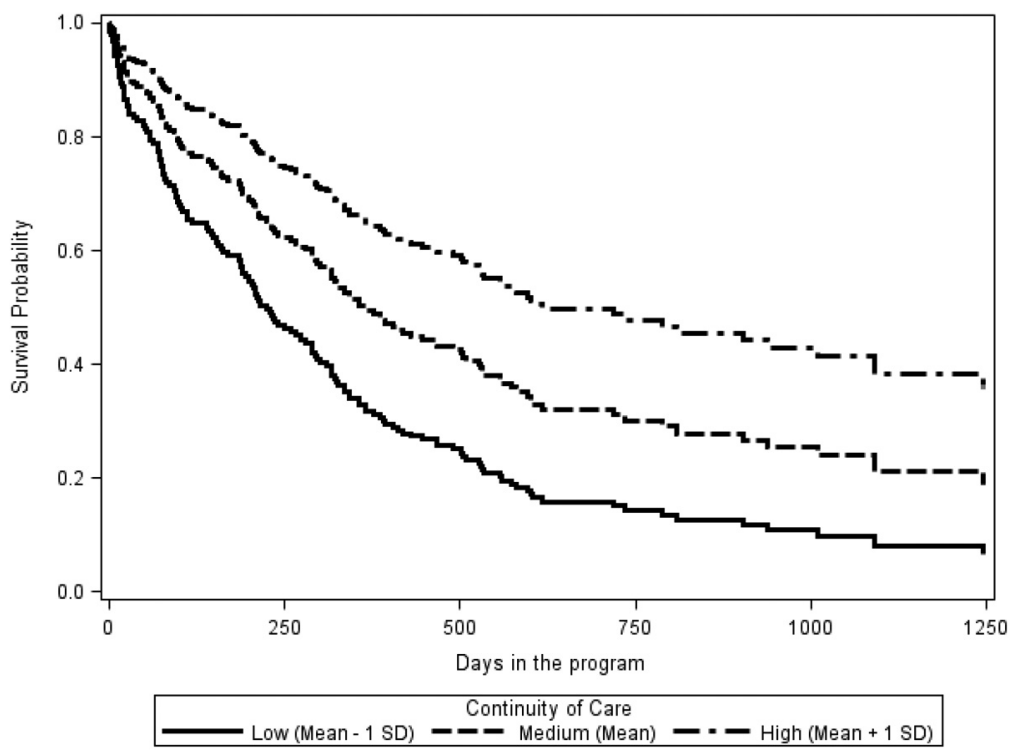

However, the dose of behavioral health contact in our study was very low (median of 1 visit), raising uncertainty as to the meaning behind this observed finding. It is possible that patients with high levels of overall motivation to start and persist in MAT treatment were also more motivated to see a behavioral health provider. Thus, it was not necessarily the behavioral health treatment provided to patients in this sample but rather a characteristic of the patients who sought out and completed visits with a behavioral health provider in the clinic that predicted treatment retention.

\section{Strengths}

A noteworthy strength of our MAT program is being housed within a family medicine residency clinic, where resident physicians are trained to effectively provide MAT. This creates a cadre of primary care physicians equipped to provide MAT at their new practice location on graduation. In this manner, access to MAT providers increases each year as new graduates enter primary care settings. Patients report satisfaction with receiving buprenorphine treatment in a primary care setting for its convenience, flexibility, and confidentiality. ${ }^{23,30}$

Additional strengths of this study include a 4year monitoring period of a large sample of patients receiving MAT in a family medicine residency clinic. Most of the research on retention in MAT programs addresses the first 6 months of treatment; our longitudinal dataset will allow examination of retention over long periods of time, which is appropriate for monitoring the treatment of chronic conditions such as OUD. Improving our understanding of predictors of retention can provide insights into which patients are at elevated risk for relapse and may benefit from a stepped level of care and more intensive services.

\section{Limitations}

This study is limited by data being drawn from a single family medicine residency clinic, the reliance on electronic health record data, and the retrospective nature of the study, which limits the ability to infer causality. There are limitations with our definition of retention and therefore with our study as it does not account for ongoing use of other illicit substances or intermittent lapses of care and it only looks at a finite length of time. Further, the definition of "retention" used in this study is visit-based, which is standard in the substance use research field. However, this outcome is limited in the sense that it does not give information about how patients are doing with respect to their addiction; it does not capture the extent to which patients had lapses 
or relapses. In addition, some patients may be misclassified as "dropped" when they may return to treatment after the study period ended. It is also not clear why some people discontinued treatment at our clinic; we attempted to gather such information from chart reviews, but this was not available for all patients. Future work is needed to determine the best treatment outcome to use in OUD research examining MAT programs, including consideration of continuous buprenorphine prescriptions or appropriate urine drug screens.

\section{Conclusions}

Overcoming the opioid epidemic will require innovative and multidisciplinary collaboration. Continuity of care and access to integrated behavioral health treatment seem to be related to higher levels of patient retention. Research is needed to better elucidate effective treatment models for behavioral health providers. Future research should explore the potentially key variable of continuity of care and examine means of fostering it in busy residency clinics. Future research should also investigate the reasons patients discontinue MAT treatment; qualitative inquiry with these patients could offer invaluable insights into the reasons for termination and means of helping the patients engage with treatment that is feasible and acceptable to them.

We would like to thank Dr. Bruce S. Liese, PhD, ABPP, Professor of Family Medicine and Psychiatry, Courtesy Professor of Clinical Psychology, University of Kansas for his contribution to our research project and manuscript.

To see this article online, please go to: http://jabfm.org/content/ 33/6/848.full.

\section{References}

1. McLellan AT, Lewis DC, O'Brien CP, Kleber HD. Drug dependence, a chronic medical illness implications for treatment, insurance, and outcomes evaluation. JAMA 2000;284:1689-95.

2. Mintzer IL, Eisenberg M, Terra M, MacVane C, Himmelstein DU, Woolhandler S. Treating opioid addiction with buprenorphine-naloxone in community-based primary care settings. Ann Fam Med 2007;5:146-50.

3. Martin SA, Chiodo LM, Wilson A. Retention in care as a quality measure for opioid use disorder. Subst Abus 2019;40:453-8.

4. Timko C, Schultz NR, Cucciare MA, Vittorio L, Garrison-Diehn C. Retention in medication- assisted treatment for opiate dependence: a systematic review. J Addict Dis 2016;35:22-35.

5. Morgan JR, Schackman BR, Leff JA, Linas BP, Walley AY. Injectable naltrexone, oral naltrexone, and buprenorphine utilization and discontinuation among individuals treated for opioid use disorder in a United States commercially insured population. J Subst Abuse Treat 2018;85:90-6.

6. Williams AR, Nunes EV, Bisaga A, et al. Developing an opioid use disorder treatment cascade: A review of quality measures. J Subst Abuse Treat 2018;91:5768.

7. Manhapra A, Petrakis I, Rosenheck R. Three-year retention in buprenorphine treatment for opioid use disorder nationally in the Veterans Health Administration. Am J Addict 2017;26:572-80.

8. Damian AJ, Mendelson T, Agus D. Predictors of buprenorphine treatment success of opioid dependence in two Baltimore City grassroots recovery programs. Addict Behav 2017;73:129-32.

9. Hser YI, Saxon AJ, Huang D, et al. Treatment retention among patients randomized to buprenorphine/naloxone compared to methadone in a multisite trial. Addiction 2014;109:79-87.

10. Marcovitz DE, McHugh RK, Volpe J, Votaw V, Connery HS. Predictors of early dropout in outpatient buprenorphine/naloxone treatment. Am J Addict 2016;25:472-7.

11. Schuman-Olivier Z, Weiss RD, Hoeppner BB, Borodovsky J, Albanese MJ. Emerging adult age status predicts poor buprenorphine treatment retention. J Subst Abuse Treat 2014;47:202-12.

12. Weinstein ZM, Kim HW, Cheng DM, et al. Longterm retention in office based opioid treatment with buprenorphine. J Subst Abuse Treat 2017;74:6570.

13. Neumann AM, Blondell RD, Azadfard M, Nathan G, Homish GG. Primary care patient characteristics associated with completion of 6-month buprenorphine treatment. Addict Behav 2013;38:2724-8.

14. Brorson HH, Ajo Arnevik E, Rand-Hendriksen K, Duckert F. Drop-out from addiction treatment: A systematic review of risk factors. Clin Psychol Rev 2013;33:1010-24.

15. Armenian SH, Chutuape MA, Stitzer ML. Predictors of discharges against medical advice from a shortterm hospital detoxification unit. Drug Alcohol Depend 1999;56:1-8.

16. Ejlertsson G, Berg S. Continuity-of-care measures: an analytic and empirical comparison. Med Care 1984;22:231-9.

17. Saultz JW. Defining and measuring interpersonal continuity of care. Ann Fam Med 2003;1:134-43.

18. Tousignant $P$, Diop M, Fournier M, et al. Validation of 2 new measures of continuity of care based on yearto-year follow-up with known providers of health care. Ann Fam Med 2014;12:559-67. 
19. Carroll KM, Weiss RD. The role of behavioral interventions in buprenorphine maintenance treatment: A review. Am J Psychiatry 2017;174:738-47.

20. Fiellin DA, Barry DT, Sullivan LE, et al. A randomized trial of cognitive behavioral therapy in primary carebased buprenorphine. Am J Med 2013;126:74.e11-7.

21. Moore BA, Barry DT, Sullivan LE, et al. Counseling and directly observed medication for primary care buprenorphine maintenance: a pilot study. J Addict Med 2012;6:205-11.

22. Weiss RD, Potter JS, Fiellin DA, et al. Adjunctive counseling during brief and extended buprenorphine-naloxone treatment for prescription opioid dependence: a 2-phase randomized controlled trial. Arch Gen Psychiatry 2011;68:1238-46.

23. Fox AD, Masyukova M, Cunningham CO. Optimizing psychosocial support during officebased buprenorphine treatment in primary care: patients' experiences and preferences. Subst Abus 2016;37:70-5.

24. Parchman ML, Pugh JA, Hitchcock Noël P, Larme AC. Continuity of care, self-management behaviors, and glucose control in patients with type 2 diabetes. Med Care 2002;40:137-44.

25. Jeffers H, Baker M. Continuity of care: Still important in modern-day general practice. Br J Gen Pract 2016;66:396-7.

26. Tammes P, Salisbury C. Continuity of primary care matters and should be protected. BMJ 2017;356: j373.

27. Van Walraven C, Oake N, Jennings A, Forster AJ. The association between continuity of care and outcomes: a systematic and critical review. J Eval Clin Pract 2010;16:947-56.

28. Saultz JW, Lochner J. Interpersonal continuity of care and care outcomes: a critical review. Ann Fam Med 2005;3:159-66.

29. Fareed A, Eilender P, Ketchen B, et al. Factors affecting noncompliance with buprenorphine maintenance treatment. J Addict Med 2014;8:345-50.

30. Barry DT, Irwin KS, Jones ES, et al. Integrating buprenorphine treatment into office-based practice: A qualitative study. J Gen Intern Med 2009;24:21825 . 